\title{
Facial Expression Analysis using Active Shape Model
}

\author{
Reda Shbib and Shikun Zhou \\ School of Engineering, University of Portsmouth, United Kingdom \\ reda.shbib@port.ac.uk,Shikun.zhou@port.ac.uk
}

\begin{abstract}
Facial expressions analysis is a vital part of the research in human-machine interaction. This chapter introduces an automatic recognition system for facial expression from a front view human face image. Obtaining an effective facial representation from initial face images is an essential phase for strong and efficient facial expression system. In this chapter we have developed a facial expression analysis system. Firstly we have tried to evaluate facial analysis base on Active shape model (ASM). In order to detect the face images, Adaboost classifier and Haar-Like feature has been adopted to achieve face detection and tracking. The ASM then has been automatically initiated in the detected face image. Then, discriminates and reliable facial feature points has been extracted applying ASM fitting technique. The geometric displacement among the projected ASM feature points coordinates and the mean shape of ASM were used to evaluate facial expression. Using support vector machine (SVM) classifier, the obtained results has reached a recognition rate of $93 \%$.
\end{abstract}

Keywords: Facial Expression Recognition, Image processing

\section{Introduction}

Generally facial expression includes rich information regarding individual's behaviour. In addition face is an indicator of human reactions and feelings; it is also an efficient and significant non-verbal interactions means between persons. According to psychologists Mehrabian [1], information transmitted by face during a communication has a $55 \%$ while the rest is divided among the voice and the language and emotional expression. This would show the significant of the facial expression during interaction between people. Thoughts and emotional states can be well expressed through facial expression. Moreover, people can, detect and realize the attitudes of others and their sensations through their facial expression. Therefore, the Facial Expression analysis has been an area of interest in the academic field which has led to numbers applications.

Facial expressions have demonstrated to be a strong tool in identifying a human emotional state and as well his response to a state or a product. Effective analysis and measurement of facial expressions is engaged in several application areas: medication, security field, video surveillance system, games, entertainment, biometric identification, but also in many behavioral science, anthropology, and psychoanalysis [2].

Improving recognition rate of facial expression systems and techniques has been an area of interest for researchers. Some research developments were focused on what types of features have to be extracted and selected and the design of classifiers. A new clustering-based technique for facial expression analysis has been introduced by (Chen and Huang, 2003). Wang et al., (2006) used LDA classifier and rate level of $83.6 \%$ has been reached on the BU-3DFE database. Employing a 2D appearance feature using Gabor-Wavelet (GW) method an average rate of $80 \%$ has been attained.

Current research development in facial expression analysis is concentrated on automatically facial expression analysis and has reached satisfactory recognition levels under some conditions and controlled circumstances. However, most of the proposed 
approaches have some limitations. The accuracy level of classification of a facial expression analysis system is limited by many issues such as the position, light conditions, resolution and human face orientation. These limits make automatic recognition of facial expressions an important research topic. Moreover, the literature has showed that some of the developed techniques have achieved a good rate of recognition, while other techniques have a low rates resulting in a satisfactory recognition rate. This chapter focuses on this problem and the developed technique has showed high rates for all recognized facial expressions

\section{Related Works}

Facial expressions recognition has been an area of interest in the last few years accompanying with the current enhancements and developments in image and computer vision field. Generally human face covers the vital information about moods and feelings. Hence, human-computer communication is extremely based on an accurate analysis of human face. Thus, the aim of automatically extracting this type of facial information has been always an area of development.

The previous investigation in facial expressions recognition field has been initiated by Ekman and Friesen in 1970s [3]. Ekman's explorations have classified human's face expressions into seven categories such as happiness, anger, sadness neutral fear, surprise, disgust, and.

Lately, the developed technique has been expanded to a Facial Action Coding System (FACS) for coding face's expressions by utilizing the facial activities which are named action units (AUs) Ekman [4].

The developments in the domain of computer vision and image -video processing in the late of 1990s have led the recognition of facial expressions to be measured as a significant subject in multimedia systems. A face model which involves 83 pre-defined features has been presented by MPEG-4 standard known as (facial definition parameters) in which the face is described in its neutral form. In addition 68 Facial Animation Parameters (FAPs) to animate human face has been introduced also by MPEG-4 standard. FAPs can be utilized human face animation and for synthesizing fundamental expressions of face. In addition FAPs can be utilized for facial expression illustration on a general face model. MPEG-4 FAPs are commonly applied in numerous of the laboratories dealing with synthesizing and analyzing facial expression [5].

Face analysis has been well examined for automatic facial expressions analysis during the last two decades, and several techniques have been developed .Generally, these techniques are categorized into two groups: Geometrical based methods [6] and appearance based methods [7]. A geometrical representation of the face is constructed by extracting the shape and the positions of facial components. A face image has been represented by 34 fiducial points in an early effort by [8]. The points has been manually selected and the coordinate of the identified points has are utilized as features. The output was a 68 dimension feature vector

Tian et al., [9] have proposed a Multi-state Face Component approach for detecting and tracking the variation of facial elements in close front face images. This approach represented facial activities by evaluating the state transitions of identical facial components. In sequence of images, the facial movements can be modelled by calculating the geometrical movement of facial feature points among the original frame and the current frame.

Another system has been developed by [10] which involves 20 face feature points which have been manually selected. Facial Action Units (AUs) were recognized by a classification of features obtained from the tracked facial points. The performed system has showed that the representation of the face based on tracking facial points has been suitable for facial expression recognition. This technique was later extended by 
implementing a full automatic facial activities recognition system that would automatically find out facial points in the original frame and analyze facial activities utilizing the best typical features which are selected by AdaBoost algorithm [6]. However, geometrical feature extraction generally needs an efficient and accurate facial feature detection and tracking. Still many challenges are facing facial feature detection and tracking in real world conditions, and since in most case it requires manual selection of features it end up with very time consuming and error prone. Thus, another techniques for facial expression based on appearance features has been introduced.

Using the appearance based facial expression features variation in face emotions such as lines, brow wrinkles, furrows are modelled by immediately using values of pixel. Independent Component Analysis (ICA) [7] and Linear Discriminant Analysis (LDA) [11] have been used for extracting facial appearance emotions from the entire face or a specific part of the face. Usually these techniques projects human face onto a subspace, locate a group of basis images, and then faces are represented as a linear combination of these images. Active Appearance Models (AAM) [12] has been used in facial expression analysis, which makes use of Principal Component Analysis (PCA) in order to model the variation shape and texture. The models are fitted to new images by changing the parameters of shape and texture within specific bounds that has been learned from a training set. In order to get an accurate and discriminative features, in [13] the author have applied Linear Discriminant Analysis (LDA) to (AAM) parameters in order to obtain facial expression images. Another approach introduced by [14] which integrates AAM with Active Shape Models (ASM) in order to minimise the average fitting error.

A Gabor Motion Energy Filters has been used by Wu et al., [15]which is capable to collect spacio-temporal information. A Representation of an image from a visual sequence has been formed by Yang and Bhanu [16]. The proposed approach involves a registration of the face to a reference image utilizing dense SIFT flow approach and then extracting appearance feature by Local Phase Quantization (LPQ).

One of the significant tasks in feature extraction is to accurately localize facial feature points. The aspect of feature extraction was a major task for many researchers in the literature as they have assumed that the facial feature has been accurately located. However, the accurate localisation of facial points has remained a challenging job. Up to date, adaptive appearance models (AAM) seems to be the most efficient technique for facial features extraction and localization which involves an automatic detection of face's contours structures and then the shape facial appearance is described by set of parameters. If we add these parameters them to a classifier, this would perform the facial expression analysis. The initial AMM method is not able to detect some features of face which involves high differences to the mean appearance of training set.

Zhao [17] applied dynamic Local Binary Pattern (LBP) in which data is extracted over the time axis. However, the proposed approach cannot deal with some situations while high variation of expression.

In order to minimize the dimensions of appearance parameters [13] have used Fisher discrimination function. The proposed approach has achieved almost same rate of recognition comparing to the initial AAM. The above-mentioned features are not related to the description and appearance of facial shape. However, the changing of spatial relation of face structures is vital to expression recognition. Moreover not all extracted features can take part in facial recognition. To enhance the recognition rate, one has to use feature selection technique to obtain the best feature set [18].

\section{Database}

The proposed approach has been applied on Extended Cohn- Kanade database [19] which is a second version of original Cohn-Kanade database (CK). $\mathrm{CK}+$ database is one 
of the wide-ranging databases in facial expression studies which involve 123 subjects and all prototypes reactions.

\section{Proposed Approach}

The proposed approach is generally involves two tasks: The first is for extracting ASM motion based a pyramid ASM model fitting method and the second for the projected motion classification obtained by applying Support Vector Machine classifiers. After the segmentation of face candidates, 68 feature points in each face are then extracted using ASM fitting technique. The system then line up three extracted feature points, eyes and nose part, to the mean shape of ASM, and ignore the other portion of the ASM against the mean face shape of ASM to estimate the geometrical dislocation information between current and mean ASM points coordinates. Then, facial expressions recognition is the obtained based on this geometrical motion using SVMs.

\subsection{Face Detection \& Image Pre-Processing}

In order to detect the face images, Adaboost classifier and Haar-Like feature [9] has been adopted to achieve face detection and tracking. Several factors of collected may affect the accuracy, efficiency and robustness of facial expression analysis system, such as contrast, illumination, size of image, too much redundant information etc. Therefore it is most vital to pre-process the taken image before starting the facial expression analysis.

As a first step the video stream is pre-processed using Gaussian mixture modelling (GMM) for image segmentation and background subtraction in order to extract the region that contain human face. Then a multi-layer classification has been adopted using Adaboost algorithm in order to obtain the precise position of candidate's faces. Figure 1 show the proposed system below.

\subsection{Haar Features}

After image processing segmentation and background subtraction we have to extract facial features in order to detect the face candidates. In this part and for very fast features evaluation we adopt face detector developed by Paul Viola et al., [20]. Viola has developed effective Haar features for face detection in 2001 which would be and effective method to differentiate between face and non-face, one of the main advantages of using Haar-like features is it's the very fast speed of its calculation. Haar features are a composition of rectangular features. As definition, it is the difference between the summations of the value of pixels of areas within the rectangle. The detection process used by Paul Viola et al. is based on the features instead of directly pixel detections. Three types of features have been used.

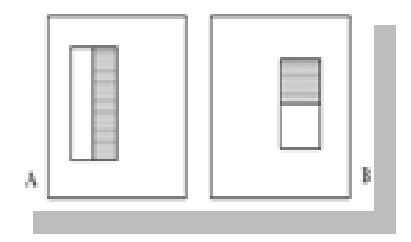

Figure 1. A: Two-rectangle Features

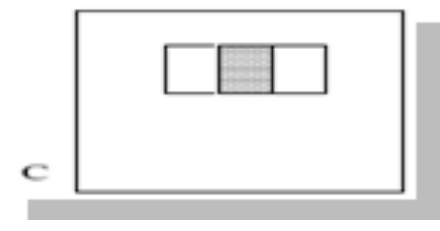

\section{C: Three Rectangle} Features

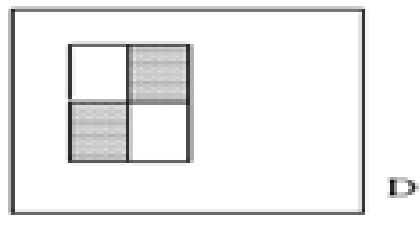

\section{D: Four- rectangle} Features

In this work, Haar features that have been selected are shown in Figure 9. 


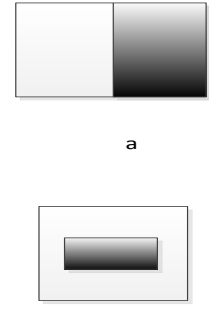

$d$

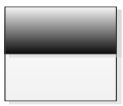

b

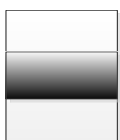

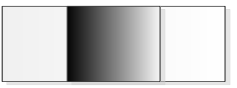

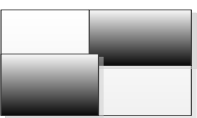

6

Figure 2. Selected Haar Features

We can calculate easily Haar features using the approach of the integral image that can be computed as follows.

$\mathrm{ii}(\mathrm{x}, \mathrm{y})=\sum_{\mathrm{x}^{\prime} \leq x y^{\prime} \leq \mathrm{y}} \mathrm{i}\left(\mathrm{x}^{\prime}, \mathrm{y}^{\prime}\right)$

Where $\mathrm{i}\left(\mathrm{x}^{\prime}, \mathrm{y}^{\prime}\right)$ represent the original image and has $\left(\mathrm{x}^{\prime}, \mathrm{y}^{\prime}\right)$ points.

Each point within the obtained integral image represents the summation of the values of pixel in the rectangle which use the origin of the image and the point in order to construct the diagonal endpoint.

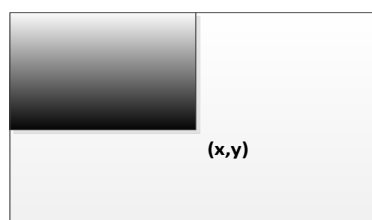

Figure 3. Integral Value of $(x, y)$

Using a recurrence process the integral image can be then calculated

$\mathrm{ii}(\mathrm{x}, \mathrm{y})=\mathrm{ii}(\mathrm{x}-1, \mathrm{y})+\mathrm{s}(\mathrm{x}, \mathrm{y})$

$\mathrm{s}(\mathrm{x}, \mathrm{y})=\mathrm{s}(\mathrm{x}, \mathrm{y}-1)+\mathrm{i}(\mathrm{x}, \mathrm{y})$

Given a set of features and tanning set of "face" and "non-face" images, there variety of machine learning technique that can be effectively used in order to learn a classification function. Adaboost techniques have been used in order to select the features set and for the classification training. Full explanation of Adaboost algorithm can be found in [20].

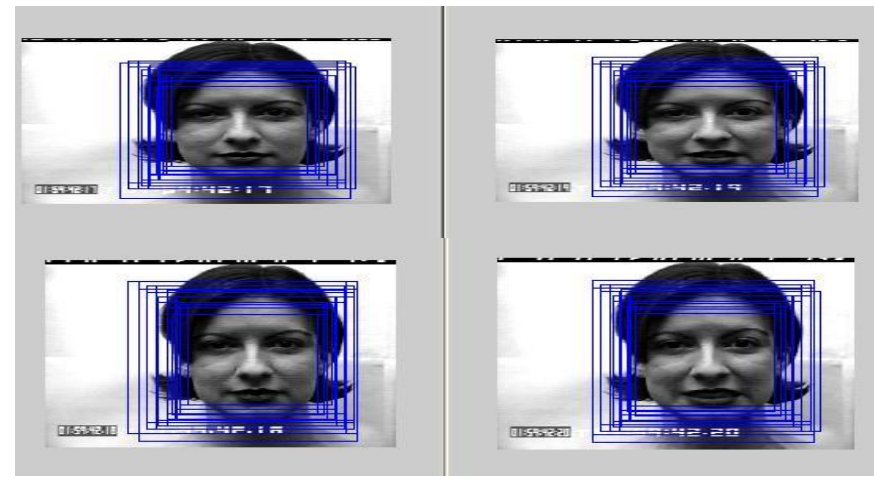

Figure 4. Detected Faces 


\subsection{Active Shape Models (ASM)}

ASMs are statistical models of the shape of objects which iteratively deform to fit to an example of the object in a new image. The shapes are formed by the point distribution model (PDM) to vary only in ways seen in a training set of labelled examples. The shape of an object is represented by a set of points which are controlled by the shape model. The ASM process tries to match the model to a new image.

Due to its flexible and reliability, ASM has the advantage over other approaches on modelling different facial features. Figure 15 shows the points of one facial expression for ASM structure.

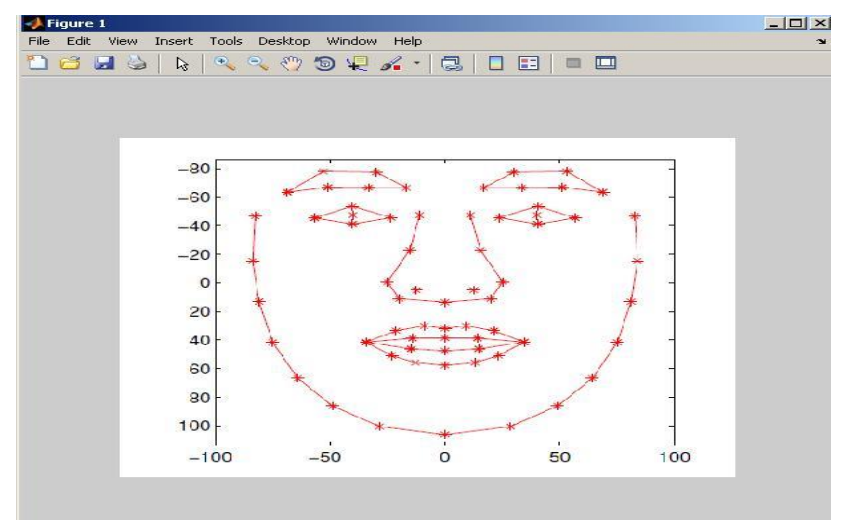

Figure 5. ASM Facial Features

Let $\mathrm{Sn}$ represent a training set which includes $\mathrm{n}$ landmark facial points. For the ith face image, $\mathrm{k}$ landmarks points are manually allocated, the coordinates of all vertices in the face image will construct the shape vector represented as follow

$v_{i}=\left[\mathrm{pt}_{\mathrm{i} 1}, \mathrm{pt}_{\mathrm{i} 2}, \ldots, \mathrm{pt}_{\mathrm{ij}}, \ldots, \mathrm{pt}_{\mathrm{ik}}\right]^{\mathrm{T}}$

Where the landmark point $\mathrm{pt}_{\mathrm{ij}}=\left(\mathrm{x}_{\mathrm{ij}}, \mathrm{y}_{\mathrm{ij}}\right)^{\mathrm{T}}$ represent the coordinates of $\mathrm{jth}$ points in the training set.

Least square technique is used to align all shapes in $\mathrm{Sn}$ into another set that contains the new transformed shapes St. Using principal component analysis (PCA) to the St set, and then we can represent $S t$ by a mean shape $\bar{s}$, the eigenvalues $\left(\gamma_{1}, \gamma_{2}, \gamma_{3}, \gamma_{n}\right)$ and their equivalent eigenvectors $\left(\mathrm{w}_{1}, \mathrm{w}_{2}, \mathrm{w}_{3}, \mathrm{~W}_{\mathrm{n}}\right)$. Select first $\mathrm{n}$ eigenvectors $\mathrm{W}=($ $\left.\mathrm{w}_{1}, \mathrm{w}_{2}, \mathrm{w}_{3}, \mathrm{w}_{\mathrm{n}}\right)$ in a such way that the vector.

$v_{\mathrm{i}}=\overline{\mathrm{s}}+\mathrm{W}_{\mathrm{a}}(5)$

Where $a=\left(a_{1}, a_{2}, \ldots, a_{n}\right)$ is the weights vectors that controlling the variants of the mean shape $\overline{\bar{s}}$ of the ASM.

In addition the profile is a significant model in ASM is. We have assumed in this work the profile to be fixed-size vector of the standard first derivative of pixel's intensity tested over the trajectory that is perpendicular to the shape contour such that the owner landmark crossing right over the middle of this profile.

For the ith landmark points in the jth training image the profile $\mathrm{P}_{\mathrm{can}}$ be obtained based on $m$ underlying image pixels. Therefore $k$ profiles $P_{i}$ is presented by $P_{i}=\left(p_{i 1}, p_{i 2}\right.$, $\ldots, \mathrm{p}_{\mathrm{ij}}$ ) for the ith landmark. 
The second-order statistics for the normalized image gradients can be then calculated. The mean profile $\overline{\mathrm{p}}_{1}$ and its corresponding covariance $\mathrm{C}_{i}$ of these profiles are then identified and considered as an important local structure for ASM. The purpose of the profile model is to improve the feature matching executions over the iterative ASM fitting process, and Mahalanobis distance between a new profile $\widehat{\mathrm{p}_{1}}$ and the associated local structure is calculated by:

$\mathrm{D}=\left(\widehat{\mathrm{p}_{1}}-\overline{\mathrm{p}}_{1}\right)^{\mathrm{T}} \mathrm{C}_{\mathrm{i}}^{-1}\left(\widehat{\mathrm{p}_{1}}-\overline{\mathrm{p}_{1}}\right)(6)$

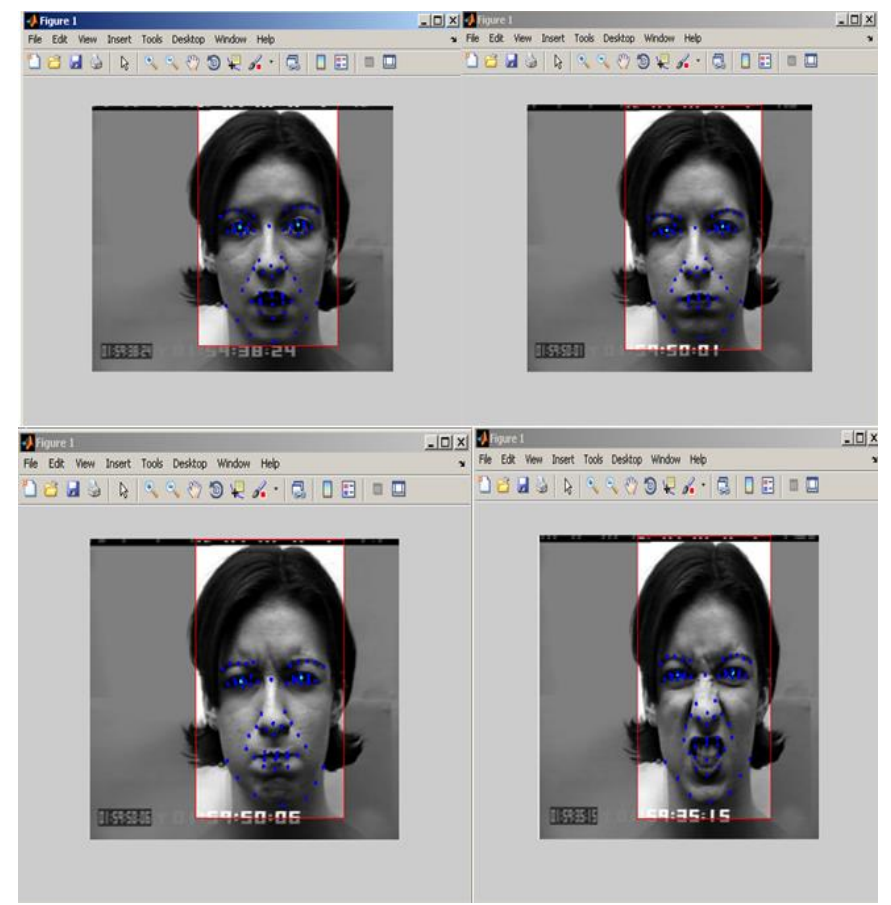

Figure 6. ASM Facial Features

\section{Active Shape Model fitting}

The proposed pyramid ASM Fitting approach showed below is described as follows:

For each level < MaxLevel, we firstly get a pyramid image img by utilizing generate pyramid image $(\cdot)$ function which down-samples the input image I; Then the proposed algorithm will iteratively calculates the relative transformation $\Delta$ (transformation $(\cdot)$ ) of $\mathrm{s}$ and original shape $\mathrm{s}_{0}$ based on minimizing the Mahalanobis distance (dis_profile $(\cdot)$ ) of every profile at each landmark point (get profile $(\cdot))$ within the pre-defined maximum repetition step (MaxRep), shape $\mathrm{s}$ is updated by invoking the update function (shape _update $(\cdot))$.

Function_AMM_Fit (I, MaxLevel, MaxRep, $\boldsymbol{s}_{0}$ )

I= Image $;$ MaxRep= number of repetition loops; $s_{0}=$ Shapes with associate profiles;

Outputs: $(\mathrm{s}, \mathrm{c}, \mathrm{t})$

$\mathrm{s}=$ shapes results;

$\mathrm{c}=$ matching cost;

$\mathrm{t}=$ transformation;

$\mathrm{s}=\mathrm{s}_{0}, \mathrm{c}=0$

for level $\leq$ MaxLevel to level $=0$

img $\leftarrow$ generate image (I, Level ) 


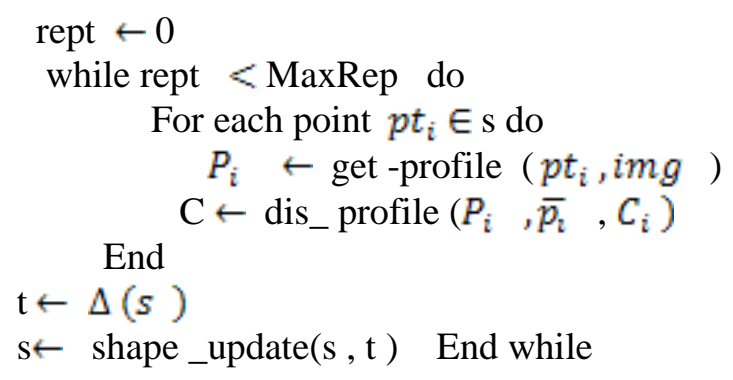

\subsection{Facial Vertices Representation}

Due to emotion of individual position and the head pose variation, the shape $\mathrm{s}$ expressing facial points has to be associated to the mean shape $\overline{\mathbf{s}}$ for the recognition task. Three facial feature points has been selected (the internal point's eyes, and the central point of nostrils which act as scare indicators shown in the fig below). The three selected fixed facial points provide the ratio of the distance over two feature points and the shape of positions while the facial expressions are changing. Assume that $\mathrm{pt}$ and $\mathrm{pt}^{y}$ relate to the same fixed facial point. In order to line up three facial points, we have applied a normalized affine transformation mechanise is described by the following as:

$$
\left[\begin{array}{cc}
\cos \alpha & -\sin \alpha \\
\sin \alpha & \cos \alpha
\end{array}\right]\left[\begin{array}{cc}
\mathrm{w}_{\mathrm{X}} & 0 \\
0 & \mathrm{w}_{\mathrm{Y}}
\end{array}\right] \mathrm{pt}+\left[\begin{array}{l}
\mathrm{t}_{\mathrm{T}_{\mathrm{X}}} \\
\mathrm{t}_{\mathrm{T}_{\mathrm{Y}}}
\end{array}\right] \text { (7) }
$$

Where, $w_{x}$ and $w_{y}$ are represent the horizontal vertical factors, $t_{r_{x}}, t_{r_{y}}$ are the translation factors and $\alpha$ is the rotation factor. After identifying these parameters applying least square technique, we can then align the complete shape s to the mean shape $\overline{\mathbf{s}}$ (Figure $16)$, converted to a new shape ${ }^{s}$.

$\mathrm{s}=\mathrm{W}^{\prime} \mathrm{s}$ where $\mathrm{W}^{t}$ is the normalizing matrix which involves the above parameters.

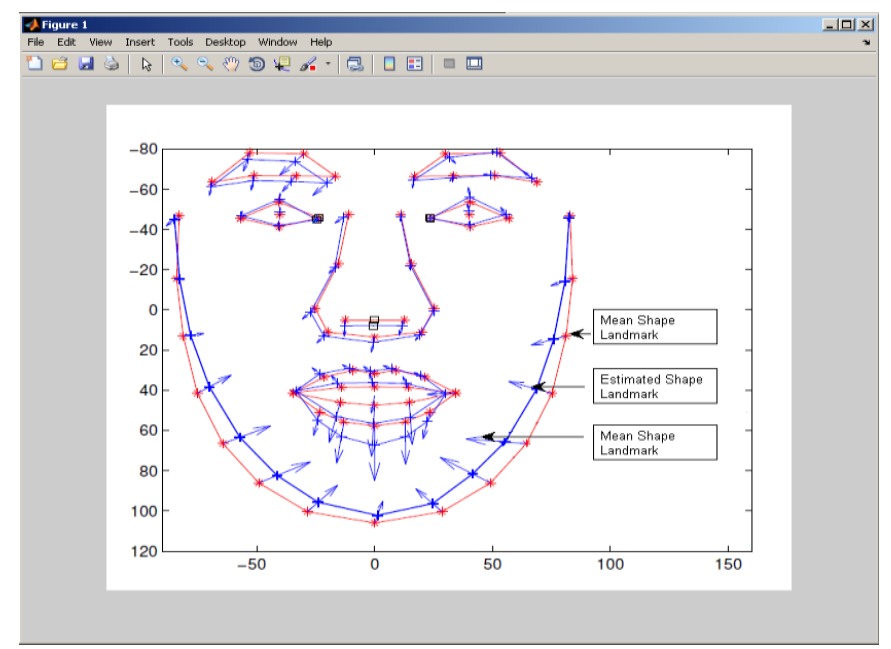

Figure 7. Shape Alignment 


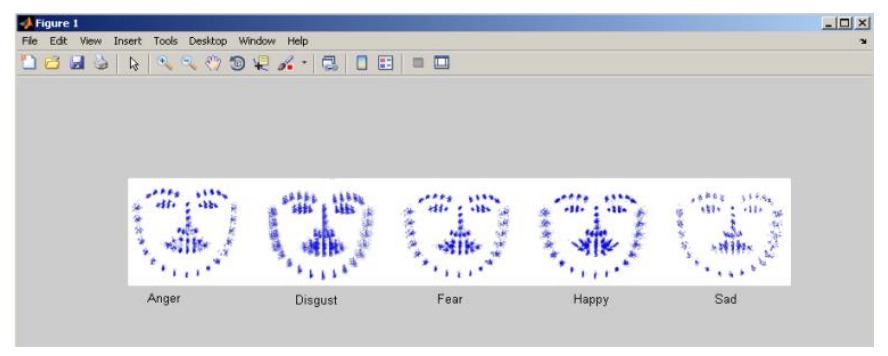

Figure 8. Estimated Shape Landmarks

In this work, the classification of facial expression depends on the geometrical information, without a direct consideration of any facial texture information. The geometrical information that has been used for facial expression analysis is the aligned shape motions with respect to the mean shape landmarks. We have described the difference $\Delta_{\tilde{\Phi}}$ of the coordinates of the entire shape nodes in the following equation

$$
\Delta_{\mathrm{s}}=\left[\omega_{\mathrm{d}} \mathrm{T}_{1}, \omega_{\mathrm{d}_{2}} \mathrm{~T}_{2}, \ldots, \omega_{\mathrm{d}} \mathrm{T}_{\mathrm{k}}\right] \mathrm{T}(8)
$$

Where $\left.\omega_{\mathrm{d}_{\mathrm{i}}=(} \quad \mathrm{pt}_{\mathrm{i}}^{\prime}-\mathrm{pt}_{\mathrm{i}}\right)^{\mathrm{T}}$ such that $\mathrm{pt}^{\prime}{ }_{\mathrm{i}} \in \mathrm{s}_{\mathrm{n}}$ and $\mathrm{pt}_{\mathrm{i}} \in \overline{\mathrm{s}}_{\text {which represents the }}$ coordinate of the new shift of the ith shape landmark. The Arrows in the figure above represent the magnification of each ${ }^{\omega_{\mathrm{d}_{\mathrm{i}}}}$

Experimental results and discussion

SVM was initially introduced for two-class classification. Given some training data D $\mathrm{D}=\left\{\left(\mathrm{X}_{\mathrm{i}}, \mathrm{Y}_{\mathrm{i}}\right) / \mathrm{X}_{\mathrm{i}}=\mathrm{d}_{\mathrm{i}}\left(\mathrm{s}_{\mathrm{i}}^{z}\right) \in \mathrm{D} / \mathrm{i}=1,2 \ldots, \mathrm{n}\right\}$

Such that $\mathrm{d}_{\mathrm{i}} \in \mathbb{K}^{\mathrm{p}}, \mathrm{Y}_{\mathrm{i}} \in\{1-.1\} / \mathrm{i}=1 \ldots \mathrm{n}$. will represent the appropriate motion of ASMs and equivalent facial classes

SVMs are obtained as resolving the quadratic programming problem as shown:

$\min _{\mathrm{w}, \mathrm{b}, \mathrm{d}_{\mathrm{i}}} \frac{\mathrm{w}^{\mathrm{T}} \mathrm{w}}{2}+\mathrm{C} \sum_{\mathrm{i}=1}^{1} \delta_{\mathrm{i}}$

This is subject to $\mathrm{Y}_{\mathrm{i}}\left[\mathrm{w}^{\mathrm{T}} \varphi\left(\mathrm{d}_{\mathrm{i}}\right)+\mathrm{b}\right] \geq 1-\delta_{\mathrm{i}}$

The training vectors $\mathrm{d}_{\mathrm{i}}$ are mapped into a higher dimensional feature space by nonlinear mapping $\varphi\left(d_{i}\right)$, then, SVM find out a linear separating hyperplane with the highest margin in order to distinct the training data in the feature space. $\mathrm{C}$ is a penalty parameter and $\mathrm{K}\left(\mathrm{d}_{\mathrm{i}} \mathrm{d}_{\mathrm{j}}\right)=\left(\mathrm{d}_{\mathrm{i}}\right) \varphi\left(\mathrm{d}_{\mathrm{j}}\right)$ represents a kernel function that describes the hyperplane.

Usually, there are two techniques for SVMs to handle the problematic of multi-class: one is to create many of two-class SVMs, for example, the "one-against-all" approach, the "one-against- one" and the "DAGSVM", The other technique would consider all data as one ideal problem. In this work, the "one-against-one" approach has been in order handle the facial expressions recognition. To efficiently distinguish between " $n$ " facial expressions it is required to train $n^{*}(n-1) / 2$ two-class SVMs. SVMs offer a specific locating of the kernel function $\mathrm{K}(\cdot)$. Linear, polynomial, are some known basic kernels. In this work, the RBF kernel (9) has been used. $\mathrm{C}$ and $\mathrm{Y}$ are chosen based on a crossvalidation. 


$$
\mathrm{K}\left(\mathrm{d}_{\mathrm{i}} \mathrm{d}_{\mathrm{j}}\right)=\mathrm{e}^{-\gamma\left\|\mathrm{d}_{\mathrm{i}}-\mathrm{d}_{\mathrm{j}}\right\|^{\mathrm{2}}}(10)
$$

We have collected only about 414 ASM emotion trials from 6 classic facial expressions and facial actions units. Essentially, additional trials can be easily produced, since ASM fitting approach is too fast and efficient enough to the head pose variation. The SVMs classification methods involving RBF kernel has need trained with a 5-fold crossvalidation technique. After training, we have obtained an optimal $\mathrm{C}=8.0$ and $\gamma=0.038$. The classification mechanism has a good performance at average 0.792 (ms). In Figure 18, some recognition expressions are presented based on shape variations. Since ASM is strong to the variation of head position, the proposed approach would identify the facial expressions with different head posture. Table 5 show the recognition rate of the proposed approach.

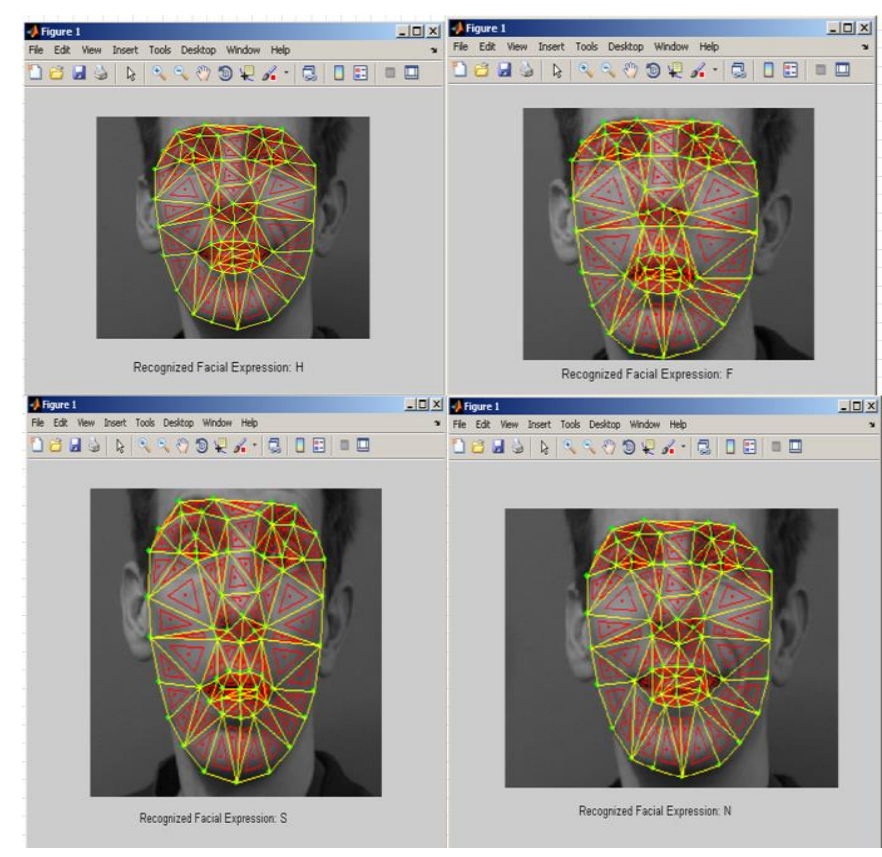

Figure 9. Facial Expressions Recognition

\begin{tabular}{lllllll}
\hline $\begin{array}{l}\text { Desired } \\
\text { Emotion }\end{array}$ & Happy & Sad & Fear & Surprise & Angry & Disgust \\
\hline Happy & 97.5 & 0.0 & 0.0 & 0.0 & 0.0 & 0.0 \\
Sad & 0.0 & 82.3 & 0.0 & 0.0 & 7.12 & 0.0 \\
Fear & 0.0 & 1.55 & 94.1 & 2.9 & 0.0 & 0.0 \\
Surprise & 0.0 & 0.0 & 4.23 & 94.3 & 0.0 & 1.46 \\
Angry & 0.0 & 2.89 & 1.44 & 0.0 & 92.74 & 2.8 \\
Disgust & 2.8 & 0.0 & 5.7 & 0.0 & 0.0 & 92.01 \\
\hline
\end{tabular}

\section{Figure 10. Recognition Performance \% of the Proposed Approach Tested for the Six Basic Emotions Using SVM Average Rate: $92.1 \%$}

We have continued our classification tests using Dynamic Bayesian Network (DBN) and Hidden Markov Model on the original CK [55] and extended CK+ [16] databases and compare our proposed method with other. 
A Dynamic Bayesian Network (DBN) is a technique in which Bayesian Network (BN) will be extended for modelling the probability distributions along semi-infinite sets of variables. Generally these variables might be divided into: $\mathrm{Gt}=\left(\mathrm{u}_{\mathrm{t}}, \mathrm{X}_{\mathrm{t}}, \mathrm{y}_{\mathrm{t}}\right)$, which represents the input, hidden layer and output variables of a state-space modelling. The $t$ term is associated with the time, in which will be increased every time when new observation is delivered.

For the verification process with our proposed approach we have utilized Cohn-Kanade Facial Expression dataset. This dataset involves 480 recordings of 97 subjects which display different facial expressions .Composed under controlled lighting and fixed background, the Cohn-Kanade dataset has been commonly used for assessing facial AUs recognition. Thus, we selected for testing use it in our work. We have taken into consideration the following AUs in our work (AU 1, 2, 3, 5, 8, 9, 11, 13, 16, 18, 19, 21, $23,24,26$, and 27).

In this method, we have modelled the relations between AUs and the matching parameters (movement on $\mathrm{x}$ and $\mathrm{y}$ axes) by applying a causal model in which the AU $\mathrm{s}$ hidden variable will cause the observations $\left(\mathrm{v}_{\mathrm{x}}, \mathrm{v}_{\mathrm{y}}\right)$, as shown in figure.

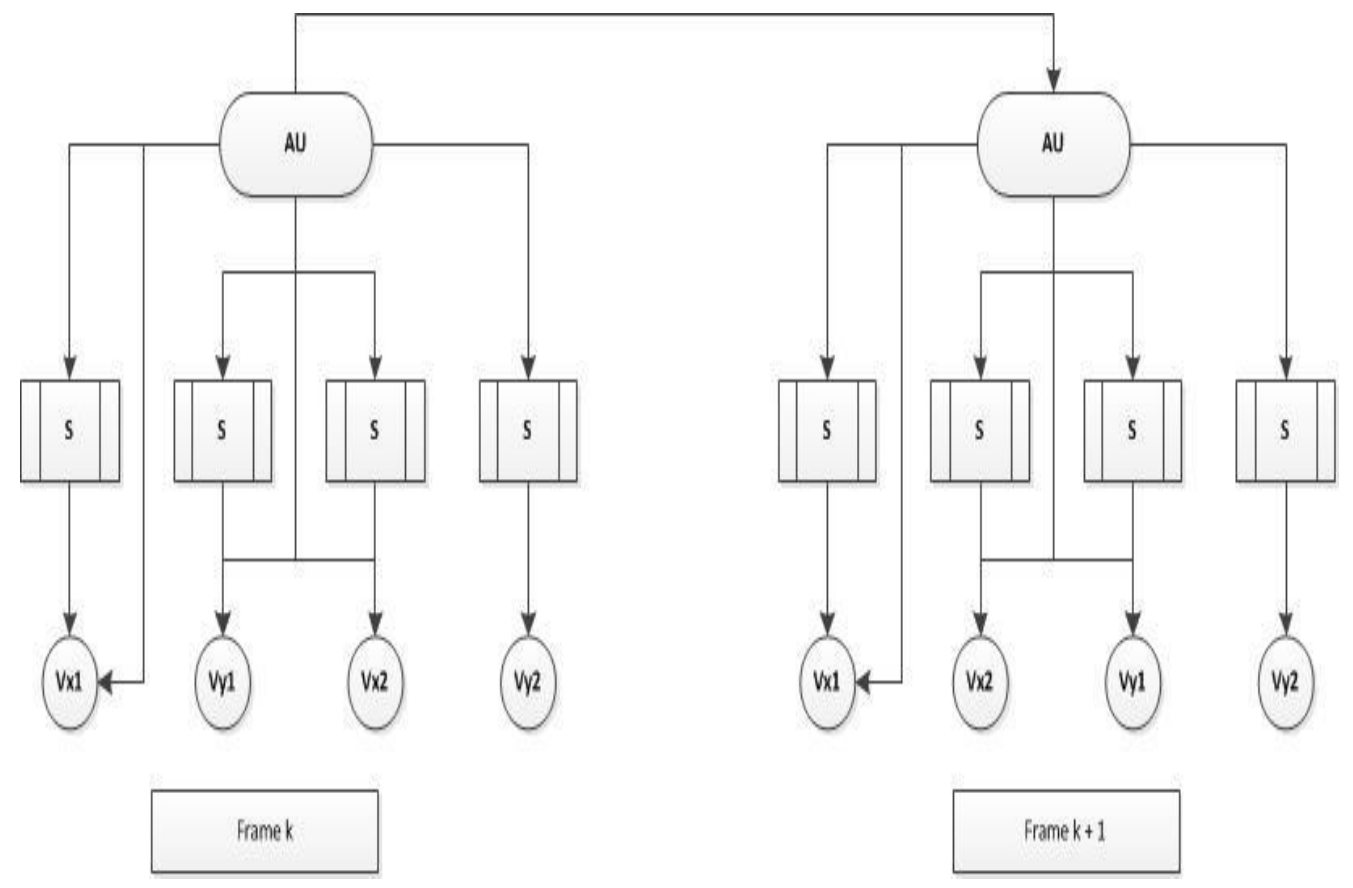

Figure 11.Dynamic Bayesian Network for AUs

In order to estimate the continuous distribution of the ${ }^{v_{x k} a n d} v_{y_{k}}$ we have applied a mixture of normal distributions, where the probability of node $S$ in each state i denotes the weight related to each mixture.

$\mathrm{P}\left(\mathrm{v}_{\mathrm{xk}} / \mathrm{AU}=\mathrm{c}\right)=\sum \mathrm{P}\left(\mathrm{v}_{\mathrm{xk}} / \mathrm{AU}=\mathrm{c}, \mathrm{S}=\mathrm{k}\right) \mathrm{P}(\mathrm{S}=\mathrm{k} / \mathrm{AU}=\mathrm{c})$ 


\begin{tabular}{lllllll}
\hline $\begin{array}{l}\text { Desired } \\
\text { Emotion }\end{array}$ & Happy & Sad & Fear & Surprize & Angry & Disgust \\
\hline Happy & 97.00 & 0.0 & 0.0 & 0.0 & 0.0 & 0.0 \\
Sad & 0.0 & 81.00 & 0.0 & 0.0 & 8.2 & 0.0 \\
Fear & 0.0 & 1.8 & 93.2 & 1.1 & 0.0 & 0.0 \\
Surprize & 1.1 & 0.0 & 3.4 & 92.6 & 0.0 & 1.21 \\
Angry & 0.0 & 2.5 & 1.7 & 0.0 & 91.3 & 2.1 \\
Disgust & 2.5 & 0.0 & 4.3 & 0.0 & 0.0 & 93 \\
\hline
\end{tabular}

Figure 12. Recognition Performance (\%) using Dynamic Bayesian Network Classifier

Average Rate: $91.35 \%$

The execution time for a pixel of size $100 * 100$ is $13.5 \mathrm{~ms}$. The figure below shows the proportional performance of execution time with Dynamic Bayesian Network classifier

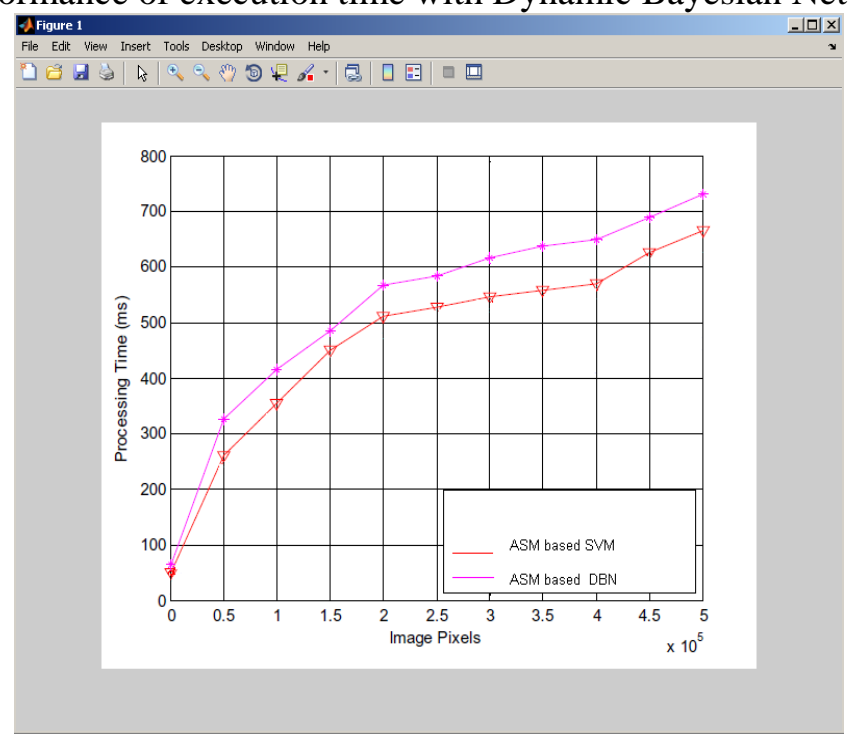

Figure 13. Processing Time Comparison using Different Classifiers

The proposed system has been compared with three different approaches in order to evaluate its performance in terms of recognition rate and execution speed as shown in table. 


\begin{tabular}{|c|c|c|}
\hline Study & Methodology & Recoginition Rate \\
\hline Proposed Method & $\begin{array}{l}\text { ASM/SVM } \\
\text { ASW/ DBN }\end{array}$ & $\begin{array}{l}92.1 \% \\
91.35 \%\end{array}$ \\
\hline (Cohen, 2000) & Dynamic programming & $60.41 \%$ \\
\hline Markov Models (Zhou et al., 2004) & Hidden Markov Models & $55.46 \%$ \\
\hline (Khanum et al., 2009) & Case-bassed reasoning & $70.83 \%$ \\
\hline Songfan (2011) & SIFT flow algointhm & $83 \%$ \\
\hline Zheng (2014) & $\begin{array}{l}\text { Group sparse reduced-rank } \\
\text { Yegression }\end{array}$ & $74 \%$ \\
\hline
\end{tabular}

Figure 14. Comparison with Different Approaches

\section{Conclusion}

Currently, facial expression recognition has become a dynamic research area. Several methods have been developed towards strong facial expression analysis, using several image acquisition, recognition and classification techniques. Facial expression analysis is an inherently multi- disciplinary domain and it is vital to look at it from all fields in order to have an insight on how to develop an efficient automatic facial expression recognition system. This work has employed several advanced methods to enhance the recognition rate and execution time of facial expression recognition system. Face detection has been carried out using the application of Viola-Jones descriptor.

Originating an effective face representation from the initial face images is an important part for an effective facial expression analysis. We have tried to evaluate ASM features in order to label the appearance variation of expression images. Extensive results has showed that ASM features are strong and reliable and for facial expression recognition. We have adopted AdaBoost to get the most discriminative facial features from a large facial feature. Best recognition rate is achieved by applying SVM classifier. However, this technique involves some limitation when it is applied to other datasets. In addition, facial features have been extracted by applying a pyramid ASM fitting technique in order to get the most discriminative facial features from large facial points. The geometrical shift among the estimated ASM feature points coordinates and mean shape of ASM is projected to SVMs classifier. Results have showed a satisfactory real-time and strong performance of the proposed approach. 


\section{References}

[1] A. Mehrabian, "Communication without words", Psychol. Today, (1986).

[2] M. Pantic and L. J. Rothkrantz, "Affect-Sensitive Multi-Modal Monitoring in Ubiquitous Computing: Advances and Challenges", ICEIS, vol. 1, (2001), pp. 466-474.

[3] P. Ekman and W. Friesen, "Pictures of Facial Affect. Consulting Psychologist", (1976).

[4] P. Ekman and W. Friesen, "The Facial Action Coding System: A Technique for the Measurement of Facial Movement", (1978).

[5] G. A. Abrantes and F. Pereira, "MPEG-4 facial animation technology: survey, implementation, and results", Circuits and Systems for Video Technology, IEEE Transactions, vol. 9, (1999), pp. 290-305.

[6] M. Valstar and M. Pantic, "Fully Automatic Facial Action Unit Detection and Temporal Analysis", Computer Vision and Pattern Recognition Workshop, 2006. CVPRW '06. Conference, (2006), pp. 149149.

[7] M. S. Bartlett, "Face recognition by independent component analysis", Neural Networks, IEEE Transactions, vol. 13, (2002), pp. 1450-1464.

[8] Z. Zhengyou, "Comparison between geometry-based and Gabor-wavelets-based facial expression recognition using multi-layer perceptron", Automatic Face and Gesture Recognition, 1998. Proceedings. Third IEEE International Conference, (1998), pp. 454-459.

[9] T. Ying-Li, "Recognizing action units for facial expression analysis", Pattern Analysis and Machine Intelligence, IEEE Transactions on, vol. 23, (2001), pp. 97-115.

[10] M. F. Valstar, "Facial Action Unit Detection using Probabilistic Actively Learned Support Vector Machines on Tracked Facial Point Data", Computer Vision and Pattern Recognition - Workshops, 2005. CVPR Workshops. IEEE Computer Society Conference, (2005), pp. 76-76.

[11] P. N. Belhumeur, "Eigenfaces vs. Fisherfaces: recognition using class specific linear projection", Pattern Analysis and Machine Intelligence, IEEE Transactions, vol. 19, (1997), pp. 711-720.

[12] T. F. Cootes, "Active appearance models", Pattern Analysis and Machine Intelligence, IEEE Transactions, vol. 23, (2001), pp. 681-685.

[13] B. Abboud, "Facial expression recognition and synthesis based on an appearance model", Signal Processing: Image Communication, vol. 19, (2004), pp. 723-740.

[14] J. Sung, "A Unified Gradient-Based Approach for Combining ASM into AAM", International journal of computer vision, vol. 75, pp. 297-309, 2007/11/01 2007.

[15] W. Tingfan, "Facial expression recognition using Gabor motion energy filters", Computer Vision and Pattern Recognition Workshops (CVPRW), 2010 IEEE Computer Society Conference, (2010), pp. 4247.

[16] Y. Songfan and B. Bhanu, "Facial expression recognition using emotion avatar image", Automatic Face \& Gesture Recognition and Workshops (FG 2011), 2011 IEEE International Conference, (2011), pp. 866-871.

[17] Z. Guoying and M. Pietikainen, "Dynamic Texture Recognition Using Local Binary Patterns with an Application to Facial Expressions", Pattern Analysis and Machine Intelligence, IEEE Transactions, vol. 29, (2007), pp. 915-928.

[18] L. Ce, "Exploring features in a Bayesian framework for material recognition", Computer Vision and Pattern Recognition (CVPR), 2010 IEEE Conference, (2010), pp. 239-246.

[19] T. Kanade, "Comprehensive database for facial expression analysis", Proceedings of the Fourth IEEE International Conference on Automatic Face and Gesture Recognition (FG'00), Grenoble, France, (2000).

[20] P. Viola and M. Jones, "Rapid object detection using a boosted cascade of simple features", Computer Vision and Pattern Recognition, 2001. CVPR 2001, Proceedings of the 2001 IEEE Computer Society Conference, vol. 1, (2001), pp. I-511-I-518. 